\title{
The structure of natural microbial enemy-victim networks
}

Timothée Poisot ${ }^{1,2,3^{*}+}$, Manon Lounnas ${ }^{1,5+}$ and Michael E Hochberg ${ }^{1,4^{*}}$

\begin{abstract}
Introduction: The persistence of generalists and specialists is a topical question in community ecology and results from both ecological and evolutionary processes. At fine taxonomical scales, ecological specialisation, i.e. organisms preferentially exploiting a subset of available habitats, is thought to be a driver promoting niche diversity. It is not clear, however, how different mechanisms interact to shape specialist-generalist coexistence.

Methods: We reconstruct the structure of five bacteria-phage networks from soil isolates, and perform an analysis of the relationships between host phylogenetic diversity, parasite specialism, and parasite performance.

Results: We show that the co-occurrence of species on a continuum of specialism/generalism is influenced by niche overlap, phage impact on bacterial hosts, and host phylogenetic structure. In addition, using a null-model analysis we show that infection strategies of the phages have more explanatory power than bacterial defenses on key structural features of these antagonistic communities.

Conclusions: We report that generalists have more impact on their hosts than specialists, even when the phylogenetic heterogeneity of hosts is controlled for. We discuss our results in the light of their implications for the evolution of biotic interactions.
\end{abstract}

Keywords: Pseudomonas fluorescens, Bipartite networks, Specialisation, Phylogeny, Food webs, Species coexistence

\section{Introduction}

Ecological specialisation is one of the central features of ecological communities (Devictor et al. 2010; Forister et al. 2012; Poisot et al. 2011a; Ravigné et al. 2009). Theoretical work identifies three broad scenarios to explain the diversity of potential or actual competitors. First, fluctuating environments may maintain competing species that otherwise could not coexist (Abrams 2006a). Second, consumers may reduce interspecific competition through foraging behaviour (Abrams 2006b, 2006c), possibly correlated with active habitat choice (Nosil et al. 2006), numerical responses (Hochberg and Hawkins 1993) or the avoidance of sub-optimal hosts or environments (Heineman et al. 2008). Finally, diversity can emerge from certain trade-off structures associated with

\footnotetext{
*Correspondence: timothee_poisot@uqar.ca; mhochber@um2.fr ${ }^{\dagger}$ Equal contributors

'Université Montpellier II, Institut des Sciences de I'Evolution, UMR 5554, Place Eugène Bataillon, 34095, Montpellier CEDEX 05, France ²Département de biologie, chimie et géographie, Université du Québec à Rimouski, 300 Allée des Ursulines, Rimouski, QC G5L 3A1, Canada Full list of author information is available at the end of the article
}

different niche exploitation strategies, although some degree of immigration from neighbouring patches is also necessary (Egas et al. 2004; Parvinen and Egas 2004).

The common thread in all of these mechanisms is that diversity is promoted when specialists exploit different niches with respect to other specialists and to generalists (Patterson et al. 2003; Ricklefs 2010; Smith et al. 2008). Past theory assumes a relationship between specificity and overall performance, i.e., fitness or population size, resulting from, for example, trade-offs between competition and parasite infection (Greenman and Hoyle 2008). Specialists can persist if, when at low abundances their growth rates are positive in the presence of competitors, which is trivially the case when their resources are not exploited by other organisms (Poisot et al. 2011a). However, all else being equal, one would expect that species evolve to become generalists if associated costs are sufficiently low and in so doing they ecologically exclude more specialised species (Dennis et al. 2011; Loxdale et al. 2011).

\section{实 Springer}


To explain the existence of highly specialised parasites, Poulin (1998) proposed the "trade-off hypothesis" $(\mathrm{TOH})$, which states that a species (such as a predator or parasite) exploiting multiple victims (prey or hosts) is more likely to encounter a range of different defence systems and, because of the costs in overcoming each, cannot be optimally adapted to all victim types. This would result in generalists being less competitive than specialists for each resource exploited, but because generalists exploit many resources they are able to coexist with specialists through across-environment (hosts) compensation.

An alternative to this is Brown's "resource breadth hypothesis" (RBH), where species with wider niches can achieve greater overall performances and are able to outcompete specialists in some habitats (Brown 1984). This latter hypothesis predicts that specialists will be competitively eliminated unless there is some form of niche differentiation, specifically if specialists have niches that do not overlap with the niche(s) of more generalist species.

Empirical evidence is consistent in some cases with $\mathrm{RBH}$ (Krasnov et al. 2004) and in others with $\mathrm{TOH}$ (Poulin 1998). Other studies report no correlation between specificity and performance (Morand and Guégan 2000), meaning that patterns of niche exploitation and differentiation do not correlate with how well species perform in their environments.

The objective of the present study is to identify the structure of antagonistic bipartite networks sampled in the field and to employ these to determine which key properties are involved in maintaining ecological diversity in these systems. The "species" in our field study are isolates of lytic bacteriophages infecting isolates of the rhizosphere bacterium Pseudomonas fluorescens. Bacteria-phage networks have advantages over other biological systems. First, they can readily provide well resolved data on performance and specificity, and thus niche breadth and overlap (Flores et al. 2011; Weitz et al. 2013). Second, they are easily sampled, and given the small spatial scales over which they typically occur (Vos et al. 2009), can be resampled to assess replicability (Koskella and Meaden 2013). Third, the strength of pairwise interactions, also called "link strengths" (Berlow et al. 2004), can easily be estimated using virulence assays, such as the negative impact of introducing a phage isolate on the population of a bacterial isolate. Finally, robust phylogenetic markers are available for P. fluorescens that enable assessment of how link strength relates to host phylogenetic diversity. This allows controlling for evolutionary inertia in defence strategies that can bias the estimate of enemy performance across its victims. This is important because the overall impact of some phages may be over- estimated if they exploit a group of phylogenetically related hosts.

We sampled five networks of $P$. fluorescens and its lytic phages along a transect in a natural environment (soil) and show that, contrary to the assumptions of $\mathrm{TOH}$ (which assumes increasing performance on the exploited niches with increased specificity), the link strengths of phages on their hosts increased with phage generality. Specialists were found to have well differentiated biotic niches, suggesting this may be a mechanism for their community persistence with generalists, thus increasing the overall diversity of host exploitation strategies. These trends were consistent across the five networks and support the idea that costs associated with the different strategies may not be the most relevant explanatory variable for interaction web and community structure in certain systems (Asplen et al. 2012; Fry 1996; Poisot et al. 2011a). We discuss the relevance of our results to the persistence of diversity of biotic niches in antagonistic associations.

\section{Methods}

\section{Isolation of bacteria and phages}

We sampled five sites along a transect in an undisturbed soil patch in Montpellier, France $\left(43^{\circ} 38^{\prime} 1.57^{\prime \prime} \mathrm{N}, 3^{\circ} 51^{\prime}\right.$ 37.60" E), on May 2, 2012. Sampling points were separated by $1,5,10$ and $20 \mathrm{~cm}$ from the first location. At each point, we sampled approximately $2 \mathrm{~g}$ of soil and from that isolated 19 arbitrary colonies of fluorescent pseudomonads (on selective Gould S1 medium; confirmed by molecular analyses) and 24 arbitrary lytic phage plaques (on lawns of P. fluorescens SBW25), to reconstruct the local interaction network following a previously described protocol (Poisot et al. 2011b). We measured the link strength for each pairwise interaction by incubating together one bacterial and one phage isolate for $24 \mathrm{~h}$ in $200 \mu \mathrm{L}$ of King's B (KB) medium at $28^{\circ} \mathrm{C}$. Link strength was used as a proxy for phage isolate performance on each host and measured as the impact on host Malthusian fitness, such that for each interaction $\mathbf{M}_{i j}$ (Lenski and May 1994)

$$
M_{i j}=1-\frac{\ln \left(N_{i j, 24} / N_{i j, 0}\right)}{\ln \left(N_{i, 24} / N_{i, 0}\right)},
$$

where $N$ is the optical density (OD) of bacteria at $0 \mathrm{~h}$ and at $24 \mathrm{~h}$ post-phage introduction, and $i$ and $j$ are respectively bacteria and phage isolates. $N_{i}$ refers to readings made of bacteria in the absence of phage, and all measures were corrected for the OD of the medium without the bacterial inoculum. Positive values of $\mathbf{M}$ indicate that the phage has a negative impact on the bacterium (presence of a link) and higher values of $\mathbf{M}$ indicate a stronger impact. Note that our estimate of $\mathbf{M}$ is 
for a single interval, and we expect that it would vary through time after phage introduction. Quantifying the temporal dynamics of phage impact and untangling the underlying ecological and evolutionary causal mechanisms could yield interesting insights but is beyond the scope of the present study. The resolution of our OD measures (Uitz et al. 2010; Zwietering et al. 1990) permits the detection of weak links, which is not always possible in other types of association, namely those that depend upon field sampling or the direct observation of rare interactions (Goldwasser and Roughgarden 1997; Olesen and Jordano 2002). Our bacteria-phage networks can be accessed online (http://dx.doi.org/10.6084/m9. figshare.696102).

\section{Measures of specificity and niche overlap}

We partition phage specificity into two indices (Bever 2002): the specificity of associations, which relates to the 'degree' of a species, i.e. the number of different bacterial hosts it exploits (Schoener 1989), and the specificity of impacts, i.e. the skew in the distribution of link strengths. These two indices are measured as follows. For each network, let $\mathbf{S}$ be a vector containing the specificity of impacts of each phage and $\mathbf{R}$ a vector containing the specificity of associations. $V$ is the number of bacterial isolates in the network and $v$ the number of bacteria on which the phage isolate under consideration has a link strength greater than 0 (i.e. its infectivity). For each phage genotype $j$, we call $P$ the vector of link strengths on all the hosts, which is normalised and sorted such that $P_{1}=1$ and $P_{1} \geq P_{n} \geq P_{V}$. The specificity of impacts is measured using the Paired Differences Index (PDI) (Poisot et al. 2012), such that

$$
S_{j}=\frac{\sum_{i=2}^{V}\left(P_{1}-P_{i}\right)}{V-1},
$$

and the specificity of associations is measured using the Resource Range (RR) index, such that

$$
R_{j}=\frac{V-v_{j}}{V-1} .
$$

This latter index is a modification of measures of species degree or generality (Schoener 1989), such that a generalist species (establishing links with all bacterial isolates) has $\mathbf{R}=0$ and a specialist (establishing a single link) has $\mathbf{R}=1$. Note that for both $R R$ and PDI a value of 1 reflects maximum specialisation. Both of these measures consider specificity on a continuum, i.e. do not discretely separate parasites into specialists (single host) and generalists (two hosts and more).

For each genotype we calculate its mean niche overlap using a measure based on quantitative link strength information (Hurlbert 1978). Niche overlap between two phages denoted by $a$ and $b$ is given by

$$
C_{a b}=1-\frac{1}{2}\left(\sum\left|\Omega_{a i}-\Omega_{b i}\right|\right)
$$

wherein $\Omega_{a i}$ is

$$
\Omega_{a i}=\frac{M_{i a}}{\sum M_{i j}},
$$

which is the proportion of the total reduction in Malthusian fitness of bacteria $i$ by phage $a$ (the same calculation applies for $\Omega_{b i}$ ).

The value of 'niche overlap' for each phage isolate is the mean of niche overlaps with each of the other phage isolates

$$
C_{a}=\sum C_{a j} \forall a \neq j
$$

In addition to these measures, we define the average link strength of each phage as the mean of all the links it establishes (i.e. the mean of all $\mathbf{P}>0$ ). The highest performance of each phage is given by $\max (\mathbf{P})$.

\section{Network analyses}

We calculated the nestedness of our networks using the Nestedness based on Overlap and Decreasing Fill (NODF) index because of its consistency and robustness (Almeida-Neto et al. 2008). NODF measures the extent to which species exploit a subset of the hosts exploited by another, more generalist species; an NODF value of 1 means that the matrix is perfectly nested and a value of 0 means that it is entirely anti-nested.

To check for the existence of modules (i.e. clusters of species that interact more among themselves than with other species, compared to a random expectation) we applied the LP-BRIM algorithm (Liu and Murata 2010), which optimises $Q_{\text {bip }}$, Barber's bipartite modularity (Barber 2007). To evaluate the reliability of the network partitions found by LP-BRIM, we measured what we call 'realised modularity' $\left(Q_{R}\right)$, i.e. the proportion of links established between species sharing a module. Values of $Q_{\mathrm{R}}$ close to 0.5 mean that an equal number of links are established within and between modules, and thus the division of the network into modules is not meaningful; values closer to 1 indicate the links are all established between species sharing the same module, and thus modularity is more important to network structure.

In addition to nestedness and modularity, we measured network connectance, i.e. the proportion of potential links that are realised, as it relates to the resistance/infectivity ranges of the bacteria and phage. Values close to 1 indicate that all possible interactions occur and values approaching 0 indicate few interactions. 
To assess the significance of these patterns, we apply four null models, each intended to separate the impact of phage and bacterial strategies on the network-level metrics (Additional file 1).

\section{DNA extraction and sequencing}

Each bacterial isolate was cultivated in $6 \mathrm{~mL} \mathrm{~KB}$ at $28^{\circ} \mathrm{C}$ for $24 \mathrm{~h}$ in $15 \mathrm{~mL}$ cylindrical plastic microcosms under constant shaking at $130 \mathrm{rpm}$ and then plated on KBagar at $28^{\circ} \mathrm{C}$ and grown for $48 \mathrm{~h}$. DNA was extracted from one arbitrarily chosen colony by diluting it in $150 \mu \mathrm{l}$ of sterilised water at $95^{\circ} \mathrm{C}$ for $5 \mathrm{~min}$ and then storing it on ice. Three polymorphic housekeeping genes were used in our analysis: $d s b A, \operatorname{rec} A$ and $f u s A$. These genes were chosen from among the 10 markers used by Frapolli and colleagues (Frapolli et al. 2007), since these three markers displayed the highest number of polymorphic sites and therefore allowed the reconstruction of a phylogeny with a good resolution. The detailed protocol used for amplification is described elsewhere (Frapolli et al. 2007). Briefly, PCRs were performed using the following cycles: initial denaturation at $94^{\circ} \mathrm{C}(150 \mathrm{~s})$ followed by $30 \mathrm{PCR}$ cycles $\left(94^{\circ} \mathrm{C}\right.$ for $30 \mathrm{~s}, 55^{\circ} \mathrm{C}$ for $30 \mathrm{~s}$ and $72^{\circ} \mathrm{C}$ for $60 \mathrm{~s}$ ) and a final extension at $72^{\circ} \mathrm{C}$ (10 min). The size of the PCR product was checked by electrophoresis on a $1 \%$ agarose gel. PCR products (amplicons) were purified and sequenced by GATC Biotech. Sequences were BLASTed against GenBank using blastx to ensure that they matched reference sequences of $P$. fluorescens. Sequences were deposited in GenBank under numbers JX139951-JX140023 (dsbA), JX140024JX140114 (fusA) and JX140115-JX140190 (recA).

\section{Phylogenetic analysis}

Alignment of DNA sequences was performed using ClustalW 2.0 (Higgins et al. 1996; Thompson et al. 1994) and manually curated before phylogenetic reconstruction. Maximum likelihood trees were inferred for each gene with PHYML v. 3.0.1 (Guindon and Gascuel 2003). The evolutionary model best fitting the data from DNA alignments was obtained using the phyml.test function for $\mathrm{R}$ 2.13.0 in ape (Paradis et al. 2004) and selected based on their Akaike Information Criterion (AIC) score. The supertree (combination of the optimal trees obtained from the analysis of individual genes) of the three genes was performed using Clann v. 3.0.0 (Creevey and McInerney 2005). These methods take into account across-gene heterogeneity in evolutionary rates by using partitionedlikelihood models, which allow each gene to evolve under a different model. Phylogenetic diversity of the bacterial isolates was measured using the PD index (Faith 1992) of all bacterial isolates infected by a given phage isolate (based on the distance within the supertree). The PDs of each phage were standardised by the largest and smallest distances in the bacterial local phylogeny, so that the phylogenetic distance across bacteria was in the interval $[0,1]$ within each sampled patch. This corrects for the fact that some sites had slightly lower bacterial phylogenetic diversity.

\section{Results}

The five networks (displayed in Additional file 2) were all highly connected $(0.76 \pm 0.10)$ and highly nested $(0.80 \pm 2.07)$ compared to random expectations, and all displayed similar structure. Additionally, using four different null models (Appendix 1), we rejected the hypothesis of random nestedness. Contrary to a previous study (Flores et al. 2011), we used a more conservative approach which accounts for the number of links established by each species (and not just the number of links in the whole network). We found a significantly higher modularity than the random expectation, which was principally due to phages (see Additional file 1).

For all networks and the system as a whole, we observed a continuum of both specificity of associations and impacts (Figure 1), which indicates that contrasting host exploitation strategies (i.e. integrating the number of host isolates, the impact achieved on each, and phylogenetic relatedness between hosts) co-occur within our communities. The values of host range, resistance and nestedness in each of the five networks were close to previous experimental findings obtained through plaquing assays (Poullain et al. 2008). There was a strong positive relationship between specificity measured as the number of bacteria upon which phages have a net negative effect and specificity measured as the differences in the intensity of negative effects, indicating that enemies with large victim-ranges tend to impact their different victims more equally than enemies with small ranges. Hereafter, we focus on specificity of impacts (i.e. skew in performances across affected victims isolates).

We found a strong negative relationship between specificity and mean link strength (Figure 2a). The same relationship was found between specificity and the strongest link of each phage (Figure 2b), indicating that highly specialised enemies do not have larger impacts on their 'optimal' victims than do generalised enemies. To account for the fact that phage impact may be similar on genetically related bacteria (one main tenet of $\mathrm{TOH}$ ), we used the normalised mean phylogenetic distance between all victims impacted by a given phage as a measure of this phage generality. We observed that generalist phages (i.e. those with greater phylogenetic distances between their victims) also had stronger impacts than relatively specialised phages (i.e. those exploiting more phylogenetically related victims; Figure 3 ).

Finally, we found a negative relationship between niche overlap and specificity, with more specialised 


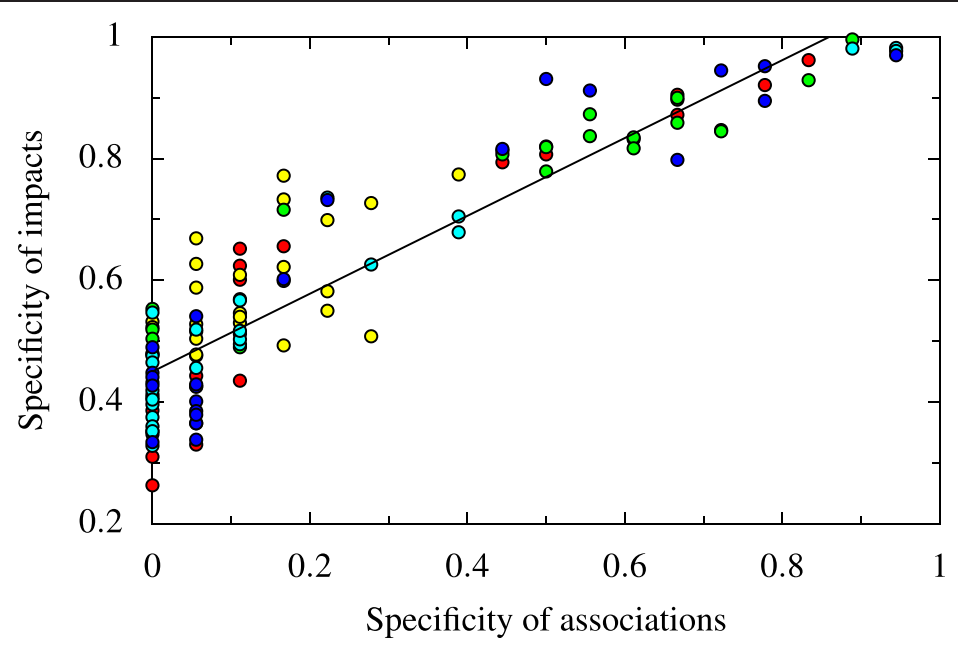

Figure 1 Relationship between the specificity of associations (as measured by RR, reflecting the proportion of available hosts exploited) and the specificity of impacts (measured by PDI, reflecting differential impact on available hosts). Both aspects of specificity are positively correlated (correlation coefficient $r=0.91, p<10^{-5}, R^{2}=0.84$ ). As for all figures, the colour of points indicates network identity. Red: $0 \mathrm{~cm}$, Blue: $1 \mathrm{~cm}$, Green: $6 \mathrm{~cm}$, Light blue: $16 \mathrm{~cm}$, Yellow: $30 \mathrm{~cm}$ from the start of the gradient.

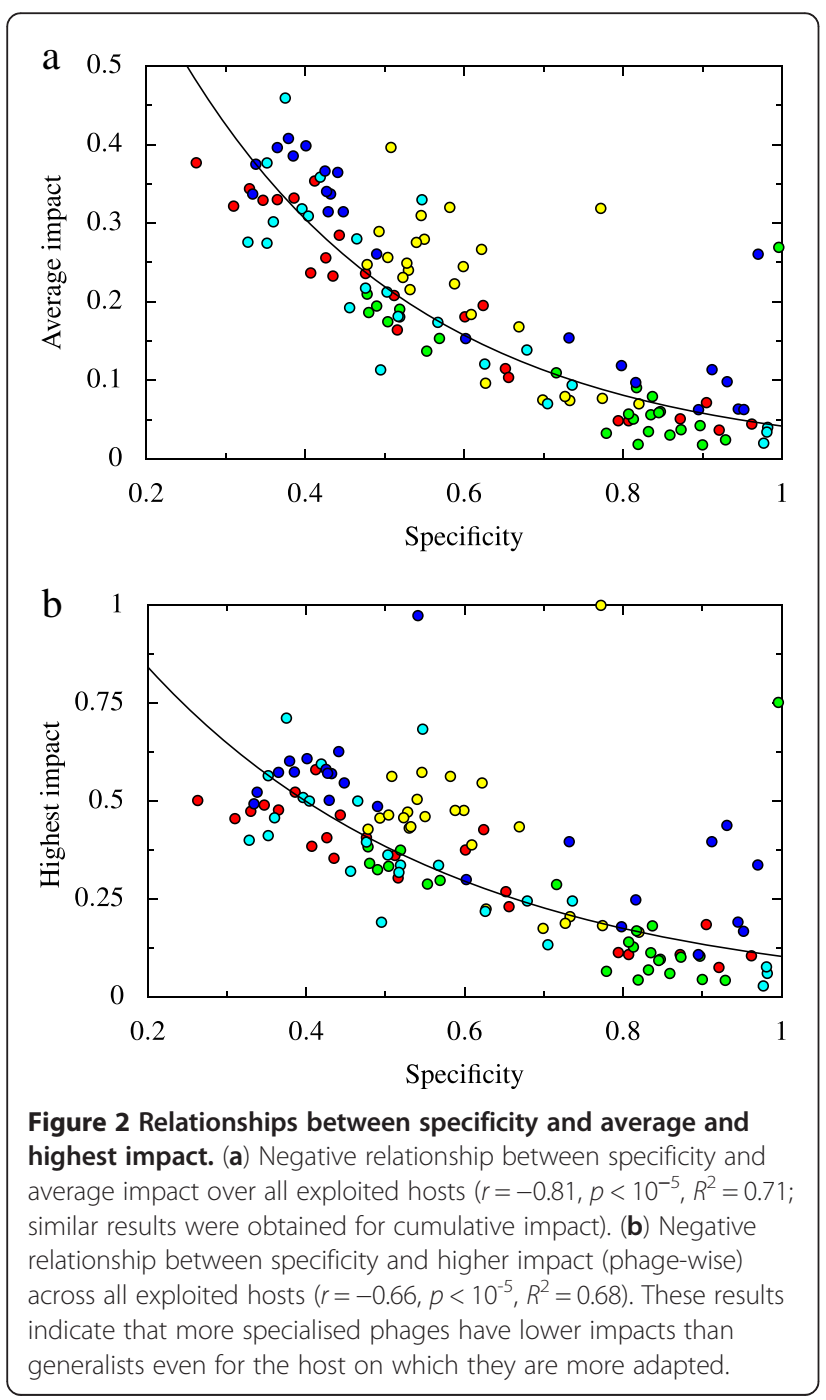

phages tending to exhibit lower overlap (Figure 4). We saw that niche overlap was relatively insensitive to specificity at low levels of the latter and strongly decreased for sufficiently high levels of specificity. While the overall trend of this relationship was similar across sites, the inflection point and plateau level were both distinctly different for Network 3. Note that Network 3 was less connected and appeared to also be more modular than the others (Table 1). This may account for the relatively lower observed niche overlap values (Figure 4) and reinforces the importance of replicating networks across time or space.

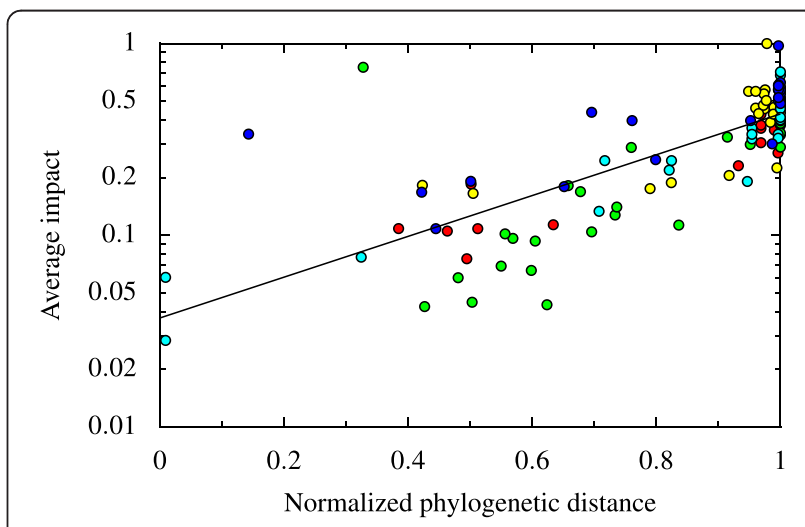

Figure 3 Positive relationship between phylogenetic distance between hosts and average impact (viewed on a decimal-log scale; $r=0.77, p<10^{-5}, R^{2}=0.61$ ). Phages that are able to attack more phylogenetically distant host isolates also have a greater impact on these hosts. 


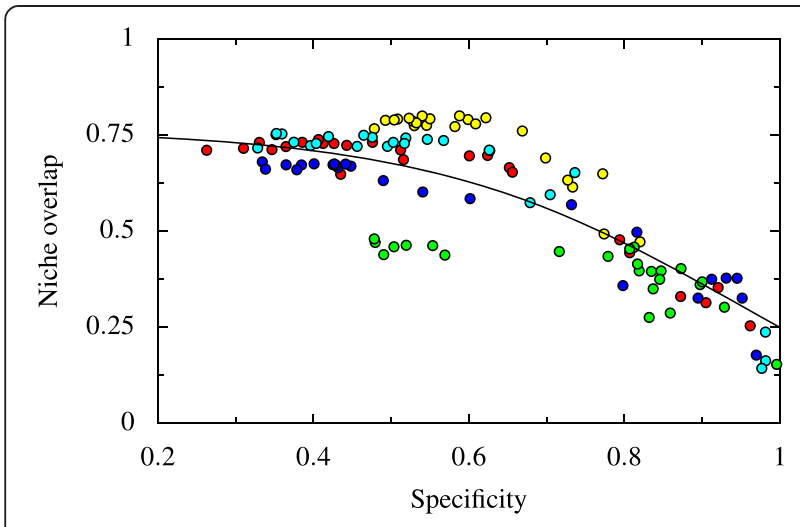

Figure 4 Negative relationship between specificity and niche overlap $\left(r=-0.81, p<10^{-5}, R^{2}=0.74\right)$. This figure suggests that the most specialised phages compete less with other isolates.

\section{Discussion}

Generalism is predicted to be costly both ecologically through the necessity of finding suitable habitats or hosts (Devictor et al. 2010; Poisot et al. 2011a) and evolutionarily due to tradeoffs with other fitness-determining traits (Bakke et al. 2002; Egas et al. 2004; Ravigné et al. 2009). The existence of such costs comes from studies of different antagonistic systems, including parasites of fishes (De Meeûs et al. 1995), birds (Fry 1990) and bacteria (Poullain et al. 2008), and of non-antagonistic systems such as pollinators (Waser et al. 1996). Based on field samples, we report that bacteriophage specialism is associated with decreased impact on bacterial isolates, both on a per-bacterium isolate and a cumulative basis. Assuming that the net impact of phages on a host is related positively to the former's fitness (Gandon and Day 2009), this suggests that specialism is costly in these bacteriophages. Recent findings on species adapting to abiotic conditions (Boulangeat et al. 2012) indicate the same trend, namely that based on measures of performance (e.g. abundance, growth rate), specialism appears to be costly or to reflect maladaptation.

The negative correlation we identified between specificity and performance could emerge if more generalised enemies exploit a larger number of closely related victims than do specialists; even if generalists and specialists have similar impacts on a given isolate, pseudo-replication due to phylogenetic similarity would inflate the cumulative impact of generalists compared to specialists (CavenderBares et al. 2009). When using the mean phylogenetic distance between hosts as a measure of specificity, we found that, for all five networks, phages had stronger impacts when exploiting the most phylogenetically dispersed bacteria (Figure 3). This indicates that, in these situations, phages tend to have stronger links with each bacterial isolate, in apparent contradiction with the predictions of the trade-off hypothesis (Poulin 1998). This result reinforces the idea that phylogenetic diversity at both trophic levels can have far-reaching consequences on the distribution, breadth and overlap of species' biotic niches (Mouquet et al. 2012). This calls for a more explicit integration of how phylogenetic structure drives interactions in network studies (Eklof et al. 2011), which will require sequencing both neutrally evolving and functional markers involved in the interaction.

Interestingly, we observed that specialism estimates are distributed along a continuum in all five networks (Figure 1), suggesting that numerous phages with different specificities may coexist. This continuum (as opposed to the persistence of only specialists or only generalists) can be explained by the observation that specialist phages tend to show less niche overlap than do generalists, suggesting decreased competition involving the former (Schoener 1974). This could explain both specialist-specialist and specialist-generalist coexistence, despite the apparent advantage of generalists in competition. Specialist phages could represent genotypes having recently immigrated from neighbouring patches (Nuismer et al. 2003) and in the process of adapting locally to environmental conditions, thus displaying lower performance. This scenario is consistent with recent data on bacteriaphage systems in natural environments (e.g. Gomez and Buckling 2011; Koskella et al. 2011; Vos et al. 2009). Were this the only factor involved, we would nonetheless expect the presence of locally adapted, high fitness specialist

Table 1 Structural metrics for the five networks studied

\begin{tabular}{|c|c|c|c|c|c|c|c|}
\hline Network & Connectance & Nestedness & Generality & Vulnerability & $Q_{\text {bip }}$ & $m$ & $Q_{\mathrm{R}}$ \\
\hline 1 & 0.80 & 82.03 & $0.80 \pm 0.25$ & $0.80 \pm 0.12$ & 0.05 & 2 & 0.58 \\
\hline 2 & 0.85 & 82.31 & $0.85 \pm 0.11$ & $0.85 \pm 0.15$ & 0.06 & 2 & 0.54 \\
\hline 3 & 0.59 & 77.28 & $0.58 \pm 0.28$ & $0.58 \pm 0.16$ & 0.15 & 2 & 0.64 \\
\hline 4 & 0.81 & 79.76 & $0.81 \pm 0.29$ & $0.81 \pm 0.12$ & - & - & - \\
\hline 5 & 0.75 & 80.56 & $0.75 \pm 0.30$ & $0.75 \pm 0.13$ & 0.08 & 2 & 0.48 \\
\hline
\end{tabular}

Values for species-level metrics are mean \pm standard deviation. Nestednesses (as measured by NODF) were higher than expected when tested against four null models (see Additional file 1). Generality (infection) and vulnerability (1 - resistance) are given as the proportions of the total number of bacteria exploited and associated exploiting phages respectively. $Q_{\text {bip }}$ and $m$ are respectively Barber's bipartite modularity score and the number of identified modules using LP-BRIM (see the 'Methods' section). $Q_{R}$ is 'realised' modularity (Poisot 2013), i.e. the proportion of links occurring between species sharing a module (values close to 0.5 indicate that half of the links are established by isolates from different modules). 
phages; although rare, we did identify such isolates in our samples (see the outliers in Figure 2a,b). We do not, however, know to what extent generalist phages may have originated from specialist niche extension onto different host isolates, as suggested by recent genomic data on bacteria-phage coevolution (Hall et al. 2010, 2011; Scanlan et al. 2011). Under this scenario evolution proceeds in two steps, with specialists first acquiring new hosts at a fitness cost which is ultimately alleviated by the spread of compensatory mutations (Agudelo-Romero et al. 2008). The endpoint of this evolutionary history would be generalist phages with no apparent fitness cost, which is consistent with our observations. These and our results are in agreement with phylogenetic analyses concluding that transitions from specialists to generalists are much more common than the reverse (Forister et al. 2012; Stireman 2005). The reduction of costs associated with generalisation through compensatory mutations provides both a mechanism for these previous results, and an explanation for the patterns we report.

An important result of our study is that specialist phages tend to exploit distinct sets of bacteria (Figure 4). This indicates that these specialists compete relatively little with other phages, which could explain their persistence even if generalists have greater impacts (and potentially higher relative fitnesses) on their victims. Such a network structure could arise, for example, if each specialist phage has specific adaptations to exploit a single, otherwise resistant bacterial type. Our results suggest that specialists may exhibit a trade-off between competitive ability and victim impact, which would allow these phages to out-compete generalists or other specialists on a limited spectrum of victims. The view that costs associated with generality are a major driver of coexistence may not be true when complex interactions between genes or genomic regions exist (Remold 2012). In some complex systems found in nature, other factors (mostly ecological) may also have stronger impacts than trade-offs (Asplen et al. 2012). Our study is limited in its implications on this point, since assays were performed under controlled laboratory conditions. However, in support of this point, a previous experiment on the same system employed in the present study showed that network structure undergoes significant changes when the only modified variable is environment quality (Poisot et al. 2011b). Future work should investigate processes explaining coexistence in the field. Specifically, there is the possibility that costs associated with either defence or infection are expressed on a per-interaction basis, in which case the heterogeneity of costs across the range of enemies/victims can be a potent driver of specialist-generalist co-occurrence.

\section{Conclusions}

Our results have implications for the evolution of bipartite network structure (Weitz et al. 2013). Recent results indicate that specialism can be one of the main drivers in the evolution of network modularity (Espinosa-Soto and Wagner 2010). Our results are consistent with this observation, since specialists were found to exploit a set of distinct niches (i.e., bacterium isolates). Additionally, phages, and not bacteria, are responsible for most of the modular structure observed (Additional file 1). The existence of modules in bacteria-phage networks has been shown at large taxonomic scales (Flores et al. 2011) and is considered to result from adaptive constraints to multiple infection pathways. Based on this and on our own findings, we suggest that specialisation that results in reduced competition with other exploiters leads to modularity in bacteria-phage bipartite networks at finer taxonomical scales. From a methodological standpoint we bring new elements to the debate about how modularity should be measured. It is striking from our results that all networks are more modular than expected (as reflected by $Q_{\text {bip }}$ ), whereas most of them actually have fewer links established than expected between species sharing a module. $Q_{R}$ thus appears to be a better index for estimating the statistical and ecological significance of a modular pattern. By crossing different null models, it is possible to assess which organisms (bacteria or phages) are involved in the emergence of network structure. This will bring new interesting results to the study of complex microbial systems.

\section{Additional files}

Additional file 1: Results of the null model analysis on the five bacteria-phage networks.

Additional file 2: Figure S1. Visualisation of the five networks sampled in this study. Hosts are on the basal trophic level. Phage isolates with colours tending toward yellow have high host ranges, whereas those tending towards red have narrow host ranges. The proximity between nodes reflects the similarity of niches, suggesting that specialists exploit different niches than both generalists and other specialists.

\section{Competing interests}

The authors declare they have no competing interests.

\section{Authors' contributions}

TP, ML and MEH designed the experiment, ML conducted the experiment, TP and ML analyzed the data, TP drafted the manuscript, all authors contributed to revisions. All authors read and approved the final manuscript.

\section{Acknowledgments}

We thank Jennifer Dunne for providing us with a copy of Network3D, Sonia Kéfi and Dominique Gravel for discussions and comments on the results, Claire Gougat-Barbera for help with the molecular biology experiments, and Joshua S. Weitz and Cesar Flores for discussions about bacteria-phage networks. TP thanks the Canadian Research Chair on Continental Ecosystems Ecology for computational support. MEH thanks the Agence National de la Recherche Scientifique [EvolStress (ANR-09-BLAN-099-01)] and the McDonnell 
Foundation (JSMF 220020294/SCS-Research Award) for financial support. TP is funded by a PBEEE-MELS post-doctoral fellowship.

\section{Author details}

'Université Montpellier II, Institut des Sciences de I'Evolution, UMR 5554, Place Eugène Bataillon, 34095, Montpellier CEDEX 05, France. ${ }^{2}$ Département de biologie, chimie et géographie, Université du Québec à Rimouski, 300 Allée des Ursulines, Rimouski, QC G5L 3A1, Canada. ${ }^{3}$ Québec Centre for Biodiversity Sciences, Stewart Biological Sciences Building, 1205 Dr. Penfield Avenue, Montréal, QC H3A 1B1, Canada. ${ }^{4}$ Santa Fe Institute, 1399 Hyde Park Road, Santa Fe, NM 87501, USA. ${ }^{5}$ MIVEGEC, UMR IRD 224 CNRS 5290 UM1-UM2, 911, Avenue Agropolis, BP 64501, 34394 Montpellier Cedex 5, France.

\section{Received: 11 February 2013 Accepted: 3 May 2013}

Published: 30 May 2013

\section{References}

Abrams PA (2006a) The prerequisites for and likelihood of generalist-specialist coexistence. Am Nat 167:329-342

Abrams PA (2006b) Adaptive change in the resource-exploitation traits of a generalist consumer: the evolution and coexistence of generalists and specialists. Evolution 60:427-439

Abrams PA (2006c) The effects of switching behavior on the evolutionary diversification of generalist consumers. Am Nat 168:645-659

Agudelo-Romero P, De la Iglesia F, Elena SF (2008) The pleiotropic cost of hostspecialization in tobacco etch potyvirus. Infect Genet Evol 8:806-814. doi:10.1016/.meegid.2008.07.010

Almeida-Neto M, Guimaraes P, Guimaraes PR, Jr, et al. (2008) A consistent metric for nestedness analysis in ecological systems: reconciling concept and measurement. Oikos 117:1227-1239. doi:10.1111/j.2008.0030-1299.16644.x

Asplen MK, Bruns E, David AS, et al. (2012) Do trade-offs have explanatory power for the evolution of organismal interactions? Evolution 66:1297-1307. doi:10.1111/j.1558-5646.2012.01573.x

Bakke T, Harris PD, Cable J (2002) Host specificity dynamics: observations on gyrodactylid monogeneans. Int J Parasitol 32:281-308. doi:10.1016/500207519(01)00331-9

Barber M (2007) Modularity and community detection in bipartite networks. Phys Rev E. doi:10.1103/PhysRevE.76.066102

Berlow EL, Neutel A-M, Cohen JE, et al. (2004) Interaction strengths in food webs: issues and opportunities. J Anim Ecol 73:585-598. doi:10.1111/j.00218790.2004.00833.x

Bever JD (2002) Negative feedback within a mutualism: host-specific growth of mycorrhizal fungi reduces plant benefit. Proc R Soc B: Biol Sci 269:2595-2601. doi:10.1098/rspb.2002.2162

Boulangeat I, Gravel D, Thuiller W (2012) Accounting for dispersal and biotic interactions to disentangle the drivers of species distributions and their abundances. Ecol Lett 15:584-593. doi:10.1111/j.1461-0248.2012.01772.x

Brown JH (1984) On the relationship between abundance and distribution of species. Am Nat 124:255-279. doi:10.1086/284267

Cavender-Bares J, Kozak KH, Fine PVA, Kembel SW (2009) The merging of community ecology and phylogenetic biology. Ecol Lett 12:693-715. doi:10.1111/j.1461-0248.2009.01314.x

Creevey CJ, Mclnerney JO (2005) Clann: investigating phylogenetic information through supertree analyses. Bioinformatics 21:390-392. doi:10.1093/ bioinformatics/bti020

De Meeûs T, Hochberg ME, Renaud F (1995) Maintenance of two genetic entities by habitat selection. Evol Ecol 9:131-138

Dennis RLH, Dapporto L, Si F, Cook LM (2011) The generalism-specialism debate: the role of generalists in the life and death of species. Biol J Linn Soc 104:725-737. doi:10.1111/j.1095-8312.2011.01789.x

Devictor V, Clavel J, Julliard R, et al. (2010) Defining and measuring ecological specialization. J Appl Ecol 47:15-25. doi:10.1111/j.1365-2664.2009.01744.x

Egas M, Dieckmann U, Sabelis MW (2004) Evolution restricts the coexistence of specialists and generalists: the role of trade-off structure. Am Nat 163:518-531

Eklof A, Helmus MR, Moore M, et al. (2011) Relevance of evolutionary history for food web structure. Proc R Soc B: Biol Sci 279:1588-1596. doi:10.1098/rspb.2011.2149

Espinosa-Soto C, Wagner A (2010) Specialization can drive the evolution of modularity. PLoS Comput Biol 6:e1000719. doi:10.1371/journal.pcbi.1000719
Faith D (1992) Conservation evaluation and phylogenetic diversity. Biol Conserv 61:1-10. doi:10.1016/0006-3207(92)91201-3

Flores CO, Meyer JR, Valverde S, et al. (2011) Statistical structure of host-phage interactions. Proc Natl Acad Sci USA 108:E288-297. doi:10.1073/ pnas. 1101595108

Forister ML, Dyer LA, Singer MS, et al. (2012) Revisiting the evolution of ecological specialization, with emphasis on insect-plant interactions. Ecology 93:981-991. doi:10.1890/11-0650.1

Frapolli M, Defago G, Moenne-Loccoz Y (2007) Multilocus sequence analysis of biocontrol fluorescent Pseudomonas spp. producing the antifungal compound 2, 4-diacetylphloroglucinol. Env Microbiol 9:1939-1955

Fry JD (1990) Trade-offs in fitness on different hosts: evidence from a selection experiment with a phytophagous mite. Am Nat 136:569-580

Fry JD (1996) The evolution of host specialization: are trade-offs overrated? Am Nat 148:84-107

Gandon S, Day T (2009) Evolutionary epidemiology and the dynamics of adaptation. Evolution 63:826-838. doi:10.1111/j.1558-5646.2009.00609.x

Goldwasser L, Roughgarden J (1997) Sampling effects and the estimation of food-web properties. Ecology 78:41. doi:10.2307/2265977

Gomez P, Buckling A (2011) Bacteria-phage antagonistic coevolution in soil. Science 332:106-109. doi:10.1126/science.1198767

Greenman JV, Hoyle AS (2008) Exclusion of generalist pathogens in multihost communities. Am Nat 172:576-584. doi:10.1086/590967

Guindon S, Gascuel O (2003) A simple, fast, and accurate algorithm to estimate large phylogenies by maximum likelihood. Syst Biol 52:696-704

Hall AR, Scanlan PD, Buckling A (2010) Bacteria-phage coevolution and the emergence of generalist pathogens. Am Nat 177:44-53. doi:10.1086/657441

Hall AR, Scanlan PD, Morgan AD, Buckling A (2011) Host-parasite coevolutionary arms races give way to fluctuating selection. Ecol Lett 14:635-642. do::10.1111/j.1461-0248.2011.01624.x

Heineman RH, Springman R, Bull JJ (2008) Optimal foraging by bacteriophages through host avoidance. Am Nat 171:E149-157. doi:10.1086/528962

Higgins DG, Thompson JD, Gibson TJ (1996) Using CLUSTAL for multiple sequence alignments. Methods Enzymol 266:383-402

Hochberg ME, Hawkins BA (1993) Predicting parasitoid species richness. Am Nat 142:671-693

Hurlbert SH (1978) The measurement of niche overlap and some relatives. Ecology 59:67-77

Koskella B, Meaden S (2013) Understanding bacteriophage specificity in natural microbial communities. Viruses 5:806-823. doi:10.3390/v5030806

Koskella B, Thompson JN, Preston GM, Buckling A (2011) Local biotic environment shapes the spatial scale of bacteriophage adaptation to bacteria. Am Nat 177:440-451. doi:10.1086/658991

Krasnov BR, Mouillot D, Shenbrot Gl, et al. (2004) Geographical variation in host specificity of fleas (Siphonaptera) parasitic on small mammals: the influence of phylogeny and local environmental conditions. Ecography 27:787-797

Lenski RE, May RM (1994) The evolution of virulence in parasites and pathogens: reconciliation between two competing hypotheses. J Theor Biol 169:253265. doi:10.1006/jtbi.1994.1146

Liu X, Murata T (2010) Community detection in large-scale bipartite networks. Trans Jpn Soc Artif Intell. 25:184-192

Loxdale HD, Lushai G, Harvey JA (2011) The evolutionary improbability of "generalism" in nature, with special reference to insects. Biol J Linn Soc 103:1-18. doi:10.1111/j.1095-8312.2011.01627.x

Morand S, Guégan J-F (2000) Distribution and abundance of parasite nematodes: ecological specialisation, phylogenetic constraint or simply epidemiology? Oikos 88:563-573. doi:10.1034/j.1600-0706.2000.880313.x

Mouquet N, Devictor V, Meynard CN, et al. (2012) Ecophylogenetics: advances and perspectives. Biol Rev Cambridge Phil Soc 87:769-785. doi:10.1111/ j.1469-185X.2012.00224.X

Nosil P, Crespi BJ, Sandoval CP, Kirkpatrick M (2006) Migration and the genetic covariance between habitat preference and performance. Am Nat 167:E6678. doi:10.1086/499383

Nuismer SL, Thompson JN, Gomulkiewicz R (2003) Coevolution between hosts and parasites with partially overlapping geographic ranges. J Evol Biol 16:1337-1345

Olesen JM, Jordano P (2002) Geographic patterns in plant-pollinator mutualistic networks. Ecology 83:2416. doi:10.2307/3071803

Paradis E, Claude J, Strimmer K (2004) APE: Analyses of Phylogenetics and Evolution in R language. Bioinformatics 20:289-290. doi:10.1093/ bioinformatics/btg412 
Parvinen K, Egas M (2004) Dispersal and the evolution of specialisation in a twohabitat type metapopulation. Theor Popul Biol 66:233-248

Patterson BD, Willig MR, Stevens RD (2003) Trophic strategies, niche partitioning, and patterns of ecological organization. In: Kunz TH, Fenton MB (ed) Bat ecology. University of Chicago Press, Chicago, pp 536-579

Poisot T (2013) An a posteriori measure of network modularity [v1; ref status: awaiting peer review, http://f1000r.es/rs]. F1000Research 2:130. doi:10.12688/ f1000research.2-130.v1

Poisot T, Bever JD, Nemri A, et al. (2011a) A conceptual framework for the evolution of ecological specialisation. Ecol Lett 14:841-851. doi:10.1111/ j.1461-0248.2011.01645.X

Poisot T, Lepennetier G, Martinez E, et al. (2011b) Resource availability affects the structure of a natural bacteria-bacteriophage community. Biol Lett 7:201-204. doi:10.1098/rsbl.2010.0774

Poisot T, Canard E, Mouquet N, Hochberg ME (2012) A comparative study of ecological specialization estimators. Methods Ecol Evol 3:537-544. doi:10.1111/j.2041-210X.2011.00174x

Poulin R (1998) Large-scale patterns of host use by parasites of freshwater fishes. Ecol Lett 1:118-128. doi:10.1046/j.1461-0248.1998.00022.x

Poullain V, Gandon S, Brockhurst MA, et al. (2008) The evolution of specificity in evolving and coevolving antagonistic interactions between a bacteria and its phage. Evolution 62:1-11. doi:10.1111/j.1558-5646.2007.00260.x

Ravigné V, Dieckmann U, Olivieri I (2009) Live where you thrive: joint evolution of habitat choice and local adaptation facilitates specialization and promotes diversity. Am Nat 174:141-169

Remold S (2012) Understanding specialism when the jack of all trades can be the master of all. Proc R Soc B: Biol Sci. doi:10.1098/rspb.2012.1990

Ricklefs RE (2010) Evolutionary diversification, coevolution between populations and their antagonists, and the filling of niche space. Proc Natl Acad Sci USA 107:1265-72. doi:10.1073/pnas.0913626107

Scanlan PD, Hall AR, Lopez-Pascua LDC, Buckling A (2011) Genetic basis of infectivity evolution in a bacteriophage. Molec Ecol 20:981-989. doi:10.1111/ j.1365-294X.2010.04903.X

Schoener TW (1974) Resource partitioning in ecological communities. Science 185:27-39

Schoener TW (1989) Food webs from the small to the large. Ecology 70:1559-1589

Smith RA, Mooney KA, Agrawal AA (2008) Coexistence of three specialist aphids on common milkweed, Asclepias syriaca. Ecology 89:2187-2196

Stireman JO (2005) The evolution of generalization? Parasitoid flies and the perils of inferring host range evolution from phylogenies. J Evol Biol 18:325-36. doi:10.1111/j.1420-9101.2004.00850.x

Thompson JD, Higgins DG, Gibson TJ (1994) CLUSTAL W: improving the sensitivity of progressive multiple sequence alignment through sequence weighting, position-specific gap penalties and weight matrix choice. Nucl Acids Res 22:4673-80

Uitz J, Stramski D, Baudoux AC, et al. (2010) Variations in the optical properties of a particle suspension associated with viral infection of marine bacteria. Limnol Oceanogr 55:2317-2330

Vos M, Birkett PJ, Birch E, et al. (2009) Local adaptation of bacteriophages to their bacterial hosts in soil. Science 325:833. doi:10.1126/science.1174173

Waser NM, Chittka L, Price MV, et al. (1996) Generalization in pollination systems, and why it matters. Ecology 77:1043-1060

Weitz JS, Poisot T, Meyer JR, et al. (2013) Phage-bacteria infection networks. Trends Microbiol 21:82-91. doi:10.1016/j.tim.2012.11.003

Zwietering MH, Jongenburger I, Rombouts FM, Van't Riet K (1990) Modeling of the bacterial growth curve. Appl Env Microbiol 56:1875-1881

doi:10.1186/2192-1709-2-13

Cite this article as: Poisot et al:: The structure of natural microbial enemy-victim networks. Ecological Processes 2013 2:13.

Submit your manuscript to a SpringerOpen ${ }^{\odot}$ journal and benefit from:

- Convenient online submission

- Rigorous peer review

- Immediate publication on acceptance

- Open access: articles freely available online

- High visibility within the field

- Retaining the copyright to your article

Submit your next manuscript at $\gg$ springeropen.com 